\title{
Aluminium can activate grapevine defence through actin remodelling
}

\author{
Ruipu Wang ${ }^{1}$, Dong Duan ${ }^{2}$, Xin Zhu ${ }^{1}$, Michael Riemann ${ }^{1}$, and Peter Nick ${ }^{1}$ \\ ${ }^{1}$ Karlsruher Institut fur Technologie \\ ${ }^{2}$ Northwest University
}

October 6, 2020

\begin{abstract}
A grapevine cell line, where actin filaments are labelled by GFP, was used to show that aluminium causes actin remodeling through activation of a NADPH oxidase in the plasma membrane, followed by activation of phytoalexin synthesis genes. Elimination of actin filaments by Latrunculin B disrupts the gene activation, inhibition of MAPK signalling by the inhibitor PD98059 as well. Interestingly, aluminum also induces transcripts for ISOCHORISMATE SYNTHASE, as well as of PR1, which are known to be responsive to salicylic acid. However, aluminium-triggered defence is not as SA responses accompanied by cell death. Also in grapevine leaf discs from two genotypes contrasting in stilbene inducibility, aluminum can induce accumulation of a central grapevine phytoalexins, the stilbene aglycone trans-resveratrol, preceded by a rapid induction of transcripts for RESVERATROL SYNTHASE and the regulating transcription factor MYB14. The amplitude of this induction reflects the general stilbene inducibility of these genotypes, indicating that the aluminum effect is not caused by unspecific toxicity, but by activation of specific signalling. The findings show that, actin filaments activate a specific branch of defence signalling acting in concert with calcium-dependent PAMP triggered immunity. This pathway links apoplastic oxidative burst through MAPK signalling with the activation of defence-related transcripts.
\end{abstract}

\section{Introduction}

Although static with respect to their location, plants live in a dynamic environment, which means that stress adaptation is crucial for their survival (Mittler, 2006). A central aspect of stress resilience is the immunity against pathogens. While plants lack the adaptable immunity, found in mammals, they have evolved two tiers of innate immunity (Jones \& Dangl, 2006). For the first tier, plants sense and respond to microbes including non-pathogens via conserved Pathogen, Microbe and Damage-Associated Molecular Patterns (PAMPs, MAMPs, or DAMPs, respectively). Binding of the eliciting molecule to specific pattern recognition receptors (PRRs triggers a broadband basal immunity (Bigeard et al. , 2015) termed as PAMP triggered immunity (PTI). Since PAMPs are essential for the survival of the pathogen, selective pressure by the host towards the loss of these treacherous molecules is buffered by the selective pressure to maintain these molecules, because they are essential. This balance of antagonistic selective pressures favoured the evolution of effectors that can inhibit PTI and re-install pathogenicity. In response, plants have acquired specific receptors recognising these effectors leading to the second tier of immune system called effector-triggered immunity (ETI), which is often specific for a certain pathogen, or even to a given strain of a pathogen (Tsuda \& Katagiri, 2010).

The recognition of PAMPs by PRRs is known to trigger calcium influx, followed by activation of mitogenactivated protein kinase (MAPK) cascades, in parallel with apoplastic oxidative burst. In consequence, transcription factors are activated that will stimulate the expression of defence genes (Withers \& Dong, 2017). Some of these events are shared with ETI (Tsuda \& Katagiri, 2010), but the relative timing seems to differ. For instance, while calcium influx precedes oxidative burst during flagellin-triggered PTI of grapevine 
cells, the temporal order of these two stress inputs is reversed after elicitation with harpin, a bacterial trigger of an ETI-like response culminating in programmed cell death (Chang \& Nick, 2012).

The two levels of immunity differ also with respect to regulation by phyto-hormones. While salicylic acid (SA) seems to activate ETI as effective strategy against biotrophic pathogens, jasmonic acid (JA) is involved in the basal defence against necrotrophic pathogens. The antagonistic role of the two phyto-hormones is reflected as antagonistic relationship of PTI versus ETI (Ramirez-Prado et al., 2018). In suspension cells from the North American grape species Vitis rupestris, JA accumulated in response to PTI triggered by flg22, but not in response to harpin, a trigger of ETI-like defence (Chang et al., 2017). It should be noted, however, that during ETI, this antagonism of JA and SA signalling can sometimes be replaced by a synergistic interaction. Activation of the SA receptors NPR3 and NPR4 inArabidopsis can also activate JA response and synthesis genes through degradation of JAZ proteins (Liu et al., 2016), a mechanism proposed to prevent the spread of necrotrophic pathogens in organs, where biotrophic pathogens have been warded off by hypersensitive cell death.

As described above, the early signalling, and also the regulation of PTI and ETI by phyto-hormones can overlap to a certain extent, leading to the question, whether there are early cellular events that are associated with the dichotomy of the two immunity layers. Comparative studies, where the responses to flg22 (triggering PTI), and harpin (a bacterial elicitor triggering an ETI-like type of defence) were compared side by side in suspension cells from either grapevine (Chang \& Nick, 2012), or tobacco BY-2 (Guan et al., 2013), suggest that rapid reorganisation of actin filaments seems to qualify as early marker for cell-death related immunity. During harpin-triggered ETI-like response, the NADPH-dependent oxidase Respiratory burst oxidase Homologue $(\mathrm{RboH})$ is activated and generates superoxide. How this primary signal is transduced into cell death and/or gene activation, has remained elusive. A few hints exist, however: A role of superoxide, and phospholipase D (PLD) for actin remodelling in response to harpin has been inferred from inhibitor studies (Chang et al. , 2015). Furthermore, pharmacological modulation of actin filaments is accompanied by elevated expression of defence genes (Qiao et al. , 2010). Modulations of actin dynamics have been shown to alter SA synthesis and signalling (Matoušková et al., 2014). Although these findings indicate that actin filaments participate in defence signalling, the functional context is far from clear. Is actin remodelling necessary and sufficient for the activation of phytoalexin genes? Is actin bundling inevitably linked with cell death, or is it possible to separate these events?

A strategy to address these questions would be to trigger actin remodeling in the absence of a pathogenrelated signal and to test, whether this would activate defence responses. Actin filaments respond to numerous intracellular or extracellular signals (reviewed in Wasteneys \& Yang 2004). In particular, abiotic stress factors that induce oxidative burst often cause actin bundling, and actin is also involved in tolerance to these factors. For instance, aluminium as abundant metal, and therefore of agricultural impact, is able to cause oxidative burst (reviewed in Panda et al. , 2009), and can induce actin bundling in tobacco seedlings (Ahad \& Nick, 2007). Moreover, tobacco mutants generated by activation tagging that are tolerant to aluminium showed constitutive bundling of actin, even in absence of aluminium as stressor (Ahad \& Nick, 2007). Therefore, we used in the current study the rationale to trigger actin bundling by $\mathrm{Al}^{3+}$. To follow actin responses, we used a grapevine cell line expressing the fluorescent actin marker GFP-AtFABD2(Akaberi et al., 2018), and we investigated the resulting defence responses in cells and plants of grapevine. We show that $\mathrm{Al}^{3+}$ causes actin reorganisation dependent on $\mathrm{RboH}$, but does not induce programmed cell death. This actin reorganisation in response to $\mathrm{Al}^{3+}$ activates genes for the synthesis of $\mathrm{SA}$ and phytoalexins supporting a model, where actin participates in a pathway involved in basal immunity by connecting the input from oxidative burst with the activation of defence genes.

\section{Materials and Methods}

Cell strains and plant material

Suspension cultures of Vitis rupestris (Seibicke et al., 2002), and a transgenic cell line of V. vinifera 'Chardonnay', expressing the fluorescent actin marker GFP-AtFABD2 (Akaberi et al., 2018), were cultivated in 
liquid medium containing $4.3 \mathrm{~g} \cdot \mathrm{L}^{-1}$ Murashige and Skoog salts (Duchefa, Haarlem, The Netherlands), $30 \mathrm{gL}^{-1}$ sucrose, $200 \mathrm{mg} \cdot \mathrm{L}^{-1} \mathrm{KH}_{2} \mathrm{PO}_{4}, 100 \mathrm{mg} \cdot \mathrm{L}^{-1}$ inositol, $1 \mathrm{mg} \cdot \mathrm{L}^{-1}$ thiamine, and $0.2 \mathrm{mg} \cdot \mathrm{L}^{-1}$ 2,4-dichlorophenoxyacetic acid (2,4-D), $\mathrm{pH} 5.8$. The cells were subcultured weekly, inoculating $6 \mathrm{ml}$ of stationary cells into $30 \mathrm{ml}$ of fresh medium in $100 \mathrm{ml}$ Erlenmeyer flasks and incubated at $27^{\circ} \mathrm{C}$ in the dark at $150 \mathrm{rpm}$ on a horizontal shaker (KS250 basic, IKA Labortechnik, Staufen, Germany). For cultivation of the transgenic actin marker line, the medium was supplemented with kanamycin $\left(50 \mathrm{mg} \cdot \mathrm{L}^{-1}\right)$. Since aluminium readily partitions into inactive complexes at neutral $\mathrm{pH}$, especially in complex media, the complex MS medium was replaced by a acidified ( $\mathrm{pH}$ 4.5) sucrose solution complemented with $3 \mathrm{mM} \mathrm{CaCl}_{2}$ (Ikegawa et al. , 2000).

The Vitis vinifera ssp. sylvestris genotype 'Hö29', and the $V$. vinifera ssp. vinifera variety 'Augster Weiss' were cultivated and collected from the grapevine germplasm collection of the Botanical Garden of the Karlsruhe Institute of Technology.

Determination of cell mortality

To determine cell viability, cells treated by $200 \mu \mathrm{M} \mathrm{AlCl} 3$ were stained at $24 \mathrm{~h}$ with Evans Blue (Gaff \& Okong'O-Ogola, 1971). Cells were transferred into a custom-made staining chamber to remove the medium, and then incubated with 2.5\% Evans Blue for 3-5 min. After washing three times with distilled water, cells were mounted on a slide and observed under a light microscope (Zeiss-Axioskop 2 FS, Differential Interference Contrast, $20 \times$ objective). Evans Blue is membrane impermeable, but can penetrate into dead cells due to the breakdown of the plasma membrane, resulting in a blue staining of the cell interior. Mortality was determined as ratio of the number of dead cells over the total number of scored cells. For each time point, 1500 cells were scored in three dependent experiments.

Stress treatments and inhibitor treatments

To impose UV-C stress, the leaf discs were irradiated for $2 \mathrm{~min}$ from a distance of $12.5 \mathrm{~cm}$ by a linear fluorescent bulb $\left(\lambda_{\max }=254 \mathrm{~nm}, 15 \mathrm{~W}\right.$, Germicidal, General Electric, Japan). To impose aluminium stress, the cells were collected and resuspended in the medium described above ( $3 \%$ sucrose, $\left.3 \mathrm{mM} \mathrm{CaCl}_{2}, \mathrm{pH} 4.5\right)$ before adding $\mathrm{AlCl}_{3}$ (Sigma, Deisenhofen) to a concentration of $200 \mu \mathrm{M}$ (Ahad \& Nick, 2007). In case of leaf discs, the treatment was conducted in petri dishes with $\mathrm{AlCl}_{3}$ freshly dissolved in distilled water $(1 \% \mathrm{w} / \mathrm{v})$. To assess the role of actin filaments, Latrunculin B as inhibitor of actin polymerisation, and phalloidin, a compound stabilising F-actin (both Sigma, Deisenhofen, Germany) were used in a final concentration of $1 \mu \mathrm{M}$ diluted from an ethanolic stock solution.. Diphenylene-iodonium chloride (DPI, Sigma-Aldrich, Deisenhofen, Germany), diluted to a final concentration of $20 \mu \mathrm{M}$ from a stock in DMSO was used to inhibit the plasma membrane based NADPH oxidase. To examine the influence of MAPK signaling, the inhibitor PD98059 targeted to mitogen-activated protein kinase kinases (MAPKKs) (Sigma-Aldrich, Deisenhofen, Germany), was dissolved in DMSO and used in a final concentration of $50 \mu \mathrm{M}$. All treatments were accompanied by solvent controls, where the maximal concentration of solvent (never exceeding $0.1 \% \mathrm{v} / \mathrm{v}$ ) used in the test samples was administered. If not stated otherwise, the treatments with aluminium or the inhibitors lasted for 2 hours. All experiments were performed at day 4 after sub-cultivation, when the culture had completed proliferation and was undergoing cell expansion. For the experiments with leaf discs, fresh, fully expanded leaves (plastochrones 4 and 5) of uniform size were used.

Visualisation and quantification of actin responses in grapevine cells

The responses of actin filaments were followed in living grapevine cells of the actin marker line $V$. vinifera 'Chardonnay' GFP-AtFABD2 by spinning disc confocal microscopy on a AxioObserver Z1 (Zeiss, Jena, Germany) inverted microscope equipped with a laser dual spinning disc scan head from Yokogawa (Yokogawa CSU-X1 Spinning Disk Unit, Yokogawa Electric Corporation, Tokyo, Japan), and a cooled digital CCD camera (AxioCamMRm; Zeiss) as described in Akaberi et al. (2018). To quantify the degree of actin aggregation, a strategy modified from Schwarzerová et al. (2002) was used. Intensity profiles were collected along a grid of equally spaced lines (four lines oriented perpendicular to the cell axis) using a line width of 10 pixels and the spline averaging option (ImageJ, https://imagej.nih.gov/ij/). The profile shows peaks and troughs, corresponding to actin bundles and non-bundled actin (either G-actin or fine filaments that are 
not optically resolved). Aggregation of actin will deplete this non-bundled actin, such that the troughs are accentuated, while the peaks will turn more prominent. This phenomenon can be quantified by calculating the standard error over the profile. This standard error can therefore be used as readout for the degree of actin aggregation: Although this strategy is robust against variations in exposure parameters such as laser power, exposure time, or exposure gain, it was made sure that all images were recorded under the same magnification and exposure time by inactivating the automatic image acquisition routine of the software (ZEN, Zeiss, Jena). Around 3-5 cells per data point were used for the quantification.

To estimate the reorganisation of actin into foci (a phenomenon that was observed after treatment with the MAPK inhibitor PD98059), a different strategy had to be used: The mean coverage of actin foci was estimated using the "analyze particle" tool of ImageJ. Images were first changed into the 8-bit BW format and then transformed into a binary image using the thresholding tool. The value for the threshold was adjusted such that the dots (that were much brighter) remained, while the filaments (that were dimmer) disappeared. To ensure that no residual filaments were selected for quantification, the circularity of the particle selection tool was set to 0.9-1 (i.e. only circular or ovoid structures were selected, while filamentous structures were excluded). To avoid that noise signals were picked up, the threshold for size selection was set to a minimum of 10 square pixels. Then, the readout "area" [in square pixels] was activated in the "set measurement" tool. After application of the "analyze particle" tool to the binary image, the results were exported into a Excel spreadsheet to determine the total area of actin organised in foci. Here, 20-25 cells per data point were used for quantification.

Analysis of extracellular $\mathrm{pH}$

Extracellular alkalinisation can be used as a rapid readout for the activation of plant immunity, because it reports the co-import of proton with calcium as an earliest known event of signalling (Felix et al., 1993), and was measured as described by Qiao et al. (2010), after pre-equilibrating the cells on an orbital shaker for around $60 \mathrm{~min}$, before adding $200 \mu \mathrm{M} \mathrm{AlCl}$ either with or without $20 \mu \mathrm{M} \mathrm{GdCl}_{3}$ as compared to a control without added compounds and a further control with $20 \mu \mathrm{M} \mathrm{GdCl}_{3}$ alone.

Analysis of gene expression

The cells were collected and shock-frozen in liquid nitrogen and ground with mortar and pestle (both heatsterilied and then precooled) before extracting total RNA using Universal RNA Purification Kit (Roboklon, Germany). In case of leaf material, total RNA was isolated using the SpectrumTM Plant Total RNA Kit (Sigma, Deisenhofen, Germany) following the protocol of the producer. Gene expression was analysed at different time points after the incubation with $200 \mu \mathrm{M} \mathrm{AlCl}$. The extracted RNA was treated with a DNA-free DNase (Roboklon, Germany) to remove potential contamination of genomic DNA. The mRNA was transcribed into cDNA using the M-MuLV cDNA Synthesis Kit (New England BioLabs; Frankfurt am Main, Germany) according to the instructions of the manufacturer.

Steady-state transcript levels of the selected genes (PAL,RS, STS , MYB14, PR1 and ICS ) were measured by quantitative real-time PCR (qRT-PCR) as described in Duan et al. (2015) using the oligonucleotide primers and PCR conditions given in Supplemental Table S1. To compare the transcript levels between different samples, the $\mathrm{C}_{t}$ values from each sample were normalised to the value for the $E \Phi-1 a$ internal standard obtained from the same sample. These normalised $\mathrm{C}_{\mathrm{t}}$ values were averaged over each technical triplicate. The difference between the $\mathrm{C}_{\mathrm{t}}$ values of the target gene $\mathrm{X}$ and those for the $E \Phi$ - $1 a$ reference $\mathrm{R}$ were calculated as follows: ${ }^{C} \mathrm{t}(X)=C_{\mathrm{t}}(X)-$ $-C_{\mathrm{t}}(R)$.The finalresultwasexpressedas $2^{-\mathrm{t}(\mathrm{X})}$.Eachexperimentwasconductedinthreebiologicalreplicates, i.e.independentex

Measurement of lipid peroxidation

Lipid peroxidation as readout for oxidative damage was determined by measuring the reaction product malone dialdehyde (MDA) according to the standard protocols by Heath \& Packer (1968) and Hodgson \& Raison (1991) with some minor adjustment for grapevine leaves: the leaves (100 mg) were shock-frozen and ground in liquid nitrogen, the powder vortexed for 45 seconds in $1 \mathrm{ml}$ of $0.1 \mathrm{M}$ phosphate buffer $(\mathrm{pH}$ 
7.4) in a 2.0-ml Eppendorf tube, centrifuged for 4 minutes at $8000 \mathrm{~g}$, and then the sediment discarded. Subsequently, $200 \mu \mathrm{L}$ of supernatant were added to a reaction mixture containing $750 \mu \mathrm{L}$ acetic acid $(20 \%$ $\mathrm{w} / \mathrm{v}$ ), $750 \mu \mathrm{L}$ 2-thiobarbituric acid (aqueous solution, $0.8 \% \mathrm{w} / \mathrm{v}$ ), $200 \mu \mathrm{L}$ Milli-Q deionised water, and 100 $\mu \mathrm{L}$ sodium dodecyl sulphate $(8.1 \% \mathrm{w} / \mathrm{v})$. An identical reaction mixture, where the supernatant from the sample was replaced by an equal volume of buffer, was used as blank. The reaction mixture was incubated for $1 \mathrm{~h}$ at $98{ }^{\circ} \mathrm{C}$, and then cooled down to room temperature. The absorbance at $535 \mathrm{~nm}$ (specific signal) and $600 \mathrm{~nm}$ (background) were recorded by an ultraviolet spectrophotometer (Uvicon, Schott, Mainz). Lipid peroxidation is then calculated as $\mu \mathrm{M}$ MDA from $\mathrm{A}_{535}$ to $\mathrm{A}_{600}$ using an extinction coefficient of $155 \mathrm{mM}^{-1}$ $\mathrm{cm}^{-1}$.

\section{Results}

Actin is bundled in response to $\mathrm{Al}^{3+}$ in grapevine cells

To address the role of actin in defence, we used aluminium as tool to induce actin remodeling based on our previous work demonstrating aluminium-dependent actin bundling in tobacco (Ahad \& Nick, 2007). We therefore tested, whether this response can also be evoked in grapevine cells. In control cells, actin was organised as meshwork of fine filaments (Figure 1A ). Between these filaments, few punctate signals were seen as well, for instance, in the perinuclear region. These punctate structures presumably represent nucleation complexes (Maisch et al., 2009). When the cells were incubated with $\mathrm{AlCl}_{3}(200 \mu \mathrm{M})$, the actin filaments had, after $2 \mathrm{~h}$, reorganised into dense bundles, often aligned with the long axis of the cell and detached from the membrane (Figure 1B ). In addition, the punctate signals had increased in abundance. To statistically validate this phenomenon, actin bundling was quantified using a quantitative image analysis strategy (Figure 1C). Compared to the control, the aluminium treatment increased the degree of bundling significantly (by more than $60 \%$ ).

In order to get insight into the base for the observed bundling, we used Latrunculin B (LatB), a drug that irreversibly sequesters G-actin and prevents F-actin assembly, such that actin polymerisation is blocked. In response to $1 \mu \mathrm{M}$ LatB $(2 \mathrm{~h})$, only short rods of actin were observed, accompanied by increased diffuse fluorescence in the cytoplasm and a higher incidence of punctate signals (Suppl. Figure S1A ). The fine filaments prevalent in control cells (Figure 1A ) were completely eliminated, such that the quantification of actin bundling produced a slightly increased value (compare white bars in Suppl. Figure S1C with those inFigure 1C ). When LatB $(1 \mu \mathrm{M})$ was administered in presence of $\mathrm{AlCl}_{3}(200 \mu \mathrm{M})$, the actin filaments were significantly longer (Suppl. Figure S1B), and the degree of actin bundling was also slightly, but significantly $(P=0.05)$, increased compared to the situation without $\mathrm{AlCl}_{3}$ (Suppl. Figure S1C ). Aluminium could rescue the effect of Latrunculin B, albeit only partially. This would indicate that a part of the actin response to aluminium is due to a reduced turnover of actin filaments.

The response of actin to $\mathrm{Al}^{3+}$ requires the activity of a NADPH oxidase

Reactive oxygen species (ROS) generated by the membrane-associated NADPH oxidase Reactive burst oxidase Homologue $(\mathrm{RboH})$ represent an important early signal in the activation of defence in grapevine (Chang \& Nick, 2012; Duan et al., 2016). We asked, therefore, whether the apoplastic oxidative burst generated by $\mathrm{RboH}-$ would be required for aluminum-dependent actin bundling (Figure 2D ). To investigate this possibility, we used diphenylene iodonium (DPI), a specific inhibitor of NADPH oxidases, to suppress the formation of apoplastic superoxide anions. When DPI was administered alone, it did not cause any significant bundling of actin (Figure 2A, Figure 2C ). However, it was able to suppress the bundling response evoked by $200 \mu \mathrm{M} \mathrm{Al}^{3+}$ (Figure 2B, Figure 2C ) demonstrating that the ROS generated by RboH are necessary for aluminium-induced actin bundling (Figure 2D ).

Genes related to the stilbene pathway are induced by $\mathrm{Al}^{3+}$ depending on actin

The previous experiment indicated that $\mathrm{RboH}$ mediates the actin response to aluminium. From our previous work, we knew already that activation of $\mathrm{RboH}$ can activate genes of the stilbene pathway (Chang et al., 2011). Therefore, we asked, whether actin is necessary for this activation. We selected phenylammonium 
lyase $(P A L)$ as the first committed step of the phenylpropanoid pathway, and two subpopulations of the stilbene synthase family, resveratrol synthases $(R S)$, and canonical stilbene synthases (STS ) as markers for the potential activation of phytoalexin genes by $\mathrm{Al}^{3+}$.

As shown in Figure 3A , Al ${ }^{3+}(200 \mu \mathrm{M}, 2$ h) significantly induced the transcripts of all three genes, a bit less pronounced for STS (around 5-fold), as compared to PAL(around 6-fold), and RS (around 7-fold). To test, whether actin filaments are necessary for this activation (Figure 3B ), we first eliminated them by Latrunculin B (Suppl. Figure S1 ), before adding aluminium. For $R S$ and $S T S$, this pretreatment completely eliminated any induction by aluminium. Also for $P A L$, this effect was observed, although, here, still a net induction of around 3-fold over the control remained. To test, whether bundling of actin was sufficient to induce phytoalexins, we probed the transcript levels of $P A L$ in response to $1 \mu \mathrm{M}$ of phalloidin administered for $2 \mathrm{~h}$ in the absence of $\mathrm{Al}^{3+}$ (Suppl. Figure S2 ). We observed a 10-fold induction, which was comparable to that seen for $\mathrm{AlCl}_{3}$ treatment (Figure 3A ). Thus, bundling of actin filaments (here obtained by phalloidin) can fully mimic the effect of $\mathrm{AlCl}_{3}$ on the induction of $P A L($ Figure $3 \mathbf{B})$.

Surprisingly, LatB, if administered in the absence of aluminium, induced a conspicuous activation of all three genes as well (Figure 3A ), which for $P A L$ and $R S$ was even exceeding the reponse obtained by aluminium. In other words: two triggers that each activated gene expression, acted antagonistically, if they were combined. This outcome was not only unexpected, but even seemingly paradox.

To understand, to what extent this induction of transcripts was linked with PTI, which is initiated by a calcium influx that can be measured as extracellular alkalinisation, we probed for potential changes of extracellular $\mathrm{pH}$ in response to $\mathrm{AlCl}_{3}$ (Suppl. Figure S3 ), however, we did not observe any alkalinisation Instead, $\mathrm{pH}$ dropped slightly, which is linked with the acidification caused by aluminium ions. Consistently, there was also no dependence on $\mathrm{GdCl}_{3}$, an inhibitor of calcium channels that in PTI can block extracellular alkalinisation (Chang \& Nick, 2012).

Transcripts of MYB14 are induced by $\mathrm{Al}^{3+}$ depending on actin

The R2R3-MYB-type transcription factor MYB14 has been shown to activate the stilbene synthase promoter (Höll et al., 2013). Later, differences in the inducibility of MYB14 promoter alleles from wild (V. sylvestris ) versus domesticated ( $V$. vinifera) grapes were shown to correlate with the stress-specific differences in the expression of stilbene synthase observed between the respective genotypes (Duan et al., 2016). Therefore, in the current study, we tested, whether MYB14 transcripts were induced by aluminium, and, if so, whether this induction was dependent on actin in the same manner as $P A L, R S$, and $S T S$. As shown in Figure4, $\mathrm{Al}^{3+}$ caused a significant induction of $M Y B 14$, albeit to a lower amplitude (only around 2-fold), as compared to $P A L, R S$ and $S T S$ (Figure 3A ) that were induced between 5-fold $(S T S)$ and 7-fold $(R S)$ This induction of MYB14 by aluminium was suppressed by pretreatment with Latrunculin B (Figure 4 ). In contrast to $P A L, R S$, and $S T S$ (Figure 3A ), Latrunculin B, if administered alone, did not cause any induction. These results demonstrate that the activation of transcription factor MYB14 is dependent on actin.

Transcripts of SA response and synthesis are induced by $\mathrm{Al}^{3+}$ depending on actin

The experiments described above showed that the aluminium induced defence-related transcripts dependending on actin. Actin remodeling is often observed as hallmark for ensuing programmed cell death. In the context of grapevine cells, this has been observed, for instance, during the responses to harpin (Qiao et al., 2010), to the stilbene aglycon resveratrol (Chang et al., 2011), or to the oxylipin derivativecis -3-hexenal (Akaberi et al. 2018). Since defence-related programmed cell death is usually deployed to encounter infection by biotrophic pathogens, a defence type commonly regulated by salicylic acid (SA), we investigated the transcription of $P R-1$ (pathogenesis related 1) (Kobayashi \& Kobayashi, 2007) as readout for SA-dependent gene expression. As shown in Figure 5A , the steady-state transcript levels for $P R 1$ went up significantly in response to $\mathrm{Al}^{3+}$, and this induction was strongly (by a factor of almost 3) suppressed by Latrunculin B (Figure 5A ). The effect of aluminium could be efficiently mimicked by Phalloidin (Figure S2 ), a compound stabilising filamentous actin (F-actin) by effectively suppressing actin dynamics. Latrunculin B alone yielded only a minor response (less than $25 \%$ of the response seen with $\mathrm{Al}^{3+}$ ). While these data would 
place $P R 1$ downstream of actin, in a similar way as the phytoalexins synthesis genes $(P A L, R S$, and $S T S$ ), and the transcriptional regulator $M Y B 14$, they also lead to the next question:

If $P R 1$ as SA responsive gene is activated by aluminium through actin-dependent signaling, there should be also a response of SA-synthesis genes. Two biosynthetic pathways, leading to SA, are known (reviewed in Chen et al., 2009) - one pathway uses cinnamonic acid as substrate, and, therefore, depends on the induction ofphenylalanine ammonium lyase $(P A L)$, while the substrate of the other pathway is isochorismate, and therefore depends on the induction of isochorismate synthase (ICS ). The induction pattern for PAL had already been tested (Figure 3A ), but since this induction might also occur in context with phytoalexins synthesis itself, it does not conclusively indicate a response of SA synthesis. We, therefore, analysed the transcript levels for ICS as well to test, whether they follow the same activation pattern as the phytoalexins genes. As shown in Figure 5B, $\mathrm{Al}^{3+}$ could induce $I C S$ transcripts significantly, although the induction was only around one fifth of that seen for PR1. This aluminium response was completely suppressed by Latrunculin B, and Latrunculin B alone did not establish the ground levels, but even decreased ICS significantly. In contrast to the pattern seen for $P R 1$ and $P A L$, phalloidin was not able to mimic aluminium with respect to the induction of of $I C S($ Figure S2 $)$.

MAPK signalling is necessary for aluminium triggered gene expression

A mitogen-activated protein kinase (MAPK) cascade is known to convey the defence signal from the plasma membrane to the nucleus, leading to changes of gene expression. This signal can be interrupted in grapevine cell cultures by the specific inhibitor PD98059, such that the activation of defence genes in response to elicitors is suppressed (Chang \& Nick, 2012). We, therefore, used the same approach to investigate, whether MAPK signalling was involved in the activation of phytoalexin genes by $\mathrm{Al}^{3+}$.

As shown in Figure 6A, the MAPK inhibitor PD98059 fully suppressed the induction of $P A L, R S$, and STStranscripts in response to aluminium. We tested then, whether this inhibitor possibly interfered with aluminium-triggered actin remodeling. We used the same experimental design as in the gene-expression experiments, i.e. the cells were treated for 30 min with PD98059, followed by additional 2 hours either in absence or presence of $\mathrm{AlCl}_{3}$. We observed that the inhibitor by itself already caused actin remodeling: In addition to a mild bundling and formation of longitudinal cables (Figure 6B ), punctate signals appeared in the perinuclear region. These perinuclear dots increased significantly after the $\mathrm{Al}^{3+}$ treatment (Figure 6C ). Although such dots had already been observed in response to aluminium alone (Figure 1), their abundance and size differed to an extent that the quality of this type of actin required a different quantification strategy scoring the coverage of these actin foci (Figure 6D, E ). We find a significant increase of these foci after combined treatment with aluminium and PD98059 as compared to PD98059 alone. While such foci were occasionally seen also in cells not treated with PD98059 (see Figure 1B for an example), their incidence was much lower in absence of this inhibitor (Figure 6E ). These experiments are congruent with a model, where MAPK signaling acts downstream of aluminium-triggered actin remodeling in response to aluminium (Figure 6F ). However, since actin foci were also observed in response to the inhibitor alone indicate a feedback of inhibited MAPK signalling (or the gene expression altered by this inhibited signalling) either to actin organisation itself or to a step upstream of actin organisation.

The effect of aluminium is not linked with cytotoxicity

Since actin bundling is often heralding programmed cell death (Chang et al., 2011), we wondered, whether treatment with $200 \mu \mathrm{M} \mathrm{Al}^{3+}$ would increase mortality, using the Evans Blue dye exclusion assay. However, we failed to see any significant difference to the mock control (Figure S4 ). Mortality was around 10\% in both cases, when scored $24 \mathrm{~h}$ after onset of the treatment. Thus, the aluminium treatment triggered actin remodeling without inducing any programmed cell death.

Differential activation of stilbene synthesis in different sylvestris chemovars

We wondered, whether the pathway responsible for aluminium-induced activation of phytoalexin synthesis is active in planta. In our previous work (Duan et al. , 2015), we could characterise two chemovars in the Wild 
European Grape (V. sylvestris ) that differed with respect to stilbene inducibility (triggered by UV light) correlated with respective differences in the steady-state transcript levels of $R S$ and STS (Duan et al. , 2015), as well as for transcript levels of their activator MYB14 (Duan et al. , 2016). We selected two genotypes representing those chemovars: The $V$. vinifera variety 'Augster Weiss' was selected as representative for the low stilbene chemovar, while the $V$. sylvestris genotype 'Hö29' belonged to the high-stilbene chemovar. First, we tested, whether induction by aluminium would reproduce the differences in transcript levels seen in the past for UV-C as trigger. This was indeed the case (Figure 7A ): both, the transcripts for MYB14 as well as those for $R S$ were induced more vigorously in Hö29 as compared to Augster Weiss. For MYB14, this difference appeared earlier (highly significant from 30 min after application of aluminium) as compared to $R S$ (highly significant only at $60 \mathrm{~min}$ after application of aluminium). We asked next, whether these differences in transcript levels would lead to a corresponding difference in the accumulation of the active stilbenetrans -resveratrol. We determined the trans -resveratrol content by HPLC $24 \mathrm{~h}$ after treatment with aluminium, and found that Hö29 accumulated more than twice of trans -resveratrol as compared to Augster Weiss (Figure 7B ). Since the induction of phytoalexins-synthesis related transcripts by aluminium had been found to depend on activation of $\mathrm{RboH}$ (Figure 3 ), we wondered, whether footprints of this oxidative burst could be detected in real plant tissues. Since reactive oxygen species will react, among other targets, with membrane lipids, an oxidative burst should lead to a transient increase of lipoxygenation, which can be detected through measuring the abundance of malone dialdehyde (MDA). In fact, we were able to detect a rapid (from $15 \mathrm{~min}$ after addition of aluminium), and transient (dissipating from $45 \mathrm{~min}$ after addition aluminium) increase of MDA levels by around 30\% in Hö29, while in Augster Weiss, the MDA levels did not show any increase (Figure $\mathbf{7 C}$ ). Thus, aluminium triggers, in the two chemovar representatives, a differential response of MDA (indicative of oxidative burst), transcripts for the stilbene regulator MYB14, the phytoalexin synthesis gene $R S$, and eventually, accumulation of the active stilbenetrans -resveratrol.

\section{Discussion}

In the current study, we provide evidence that remodelling of actin filaments (in our experiments induced by aluminium-dependent activation of the NADPH oxidase $\mathrm{RboH}$ ) is sufficient to induce phytoalexins synthesis genes in grapevine. This activation of defence genes shares the dependence on MAPK signalling with flg22 triggered basal immunity, while being independent of calcium influx. The remodelling of actin is shared with cell-death related defence responses. However, this aluminium triggered defence response is not accompanied by cell death. Instead, aluminium also activates isochorismate synthase, a key enzyme for the synthesis of salicylic acid, as well as of $P R 1$, a gene which is known to be responsive to salicylic acid. In the following, we will discuss these findings in the context of a third defence pathway that bifurcates from cell-death related immunity (ETI) and merges into basal immunity (PTI), we will revisit the role of salicylic acid for basal immunity, and we will try to draw some conclusions on the nature and mechanisms of actin remodelling triggering this pathway, and give an outlook on potential applications for viticulture that can be derived from the current findings.

Actin-dependent and PTI signalling merge between calcium influx and MAPK

Activation of calcium influx is a hallmark for PTI (Gómez-Gómez \& Boller, 2002). However, while aluminium can trigger phytoalexin-synthesis transcripts, it does not produce any extracellular alkalinisation (Supp. Figure S3 ), indicating that this transcript induction is independent of calcium influx. On the other hand, in presence of the specific MAPK inhibitor PD98059, the transcript response to aluminium is blocked completely (Figure 6A ). Thus, the most straightforward working model would place the merge the merge between actin-dependent and PTI signalling downstream of calcium influx, but upstream of MAPK activation. This model leads to two implications that are both supported by the literature record: First, MAPK signalling should be activated by oxidative burst. In fact, this has been reported for a number of cases (for a recent review see Liu \& He, 2017), for instance mediated by the serine-threonine kinase OXI1 (Rentel et al. , 2004). Second, the MAPK cascade should be activated by modulations of actin dynamics. Also this implication has been experimentally confirmed (reviewed in Šamaj et al. , 2004). For instance, the activity of SIMK and SAMK, a MAP kinase from alfalfa were activated, when actin filaments were eliminated by Latrunculin 
B (Šamaj et al. , 2002; Sangwan et al. , 2002), in case of SIMK, also suppression of actin turnover by Jaspaklinolide was activating (Šamaj et al. , 2002). These authors also had observed that a MEK1 inhibitor induced remodelling of plant actin, indicative of a feedback of MAPK signalling upon actin (consistent with our findings using PD98059,Figure 6 ).

Defence-related actin remodeling can be separated from defence-related cell-death

Actin remodelling has been recognised as early event heralding ensuing programmed cell death (reviewed in Gourlay \& Ayscough, 2005; Franklin-Tong \& Gourlay, 2008; Smertenko \& Franklin-Tong, 2011). In grapevine cells, as well, the induction of programmed cell death, by either the bacterial elicitor harpin (Chang et al. , 2015; Qiao et al. , 2010), the stilbene aglycone resveratrol (Chang et al. , 2011), or the oxylipin derivative 3-cis -hexenal (Akaberi et al. , 2018) were preceded by actin remodelling, involving a depletion of the fine subcortical meshwork and massive bundling of transvacuolar actin cables. Thus, in these cases, actin remodelling was tightly correlated with programmed cell death. A correlation of two events is a necessary condition for claiming a causal relation between these two events. However, even if this correlation is tight, it cannot be considered as sufficient for causality.

In fact, actin remodelling of a similar type can be observed without any correlation with cell death. For instance, depletion of cortical actin and condensation of actin cables have been also observed in response to auxin depletion (Waller et al. , 2002), or in response to activation of the plant photoreceptor phytochrome (Waller \& Nick, 1997), both events that occur in the absence of any cell death.

The same conclusion is reached by our observation that aluminium did not cause any significant increase of mortality (Suppl. Figure S4 ). Is actin remodelling just a side phenomenon, then? This seems not to be the case either, because in the same cell system ( $V$. rupestris ), the induction of programmed cell death by harpin had been shown to be significantly mitigated by pretreatment with Latrunculin B (Chang et al. , 2015), which provides evidence that actin is necessary to activate cell death.

Thus, while actin remodelling is, usually, accompanied by defence-related cell death, and while suppression of actin remodelling will impair defence-related cell death, this apparently tight link is uncoupled in case of aluminium treatment. The most straightforward explanation would be that actin remodelling has to coincide with a second signal to activate programmed cell death. This second signal cannot be the apoplastic oxidative burst generated by $\mathrm{RboH}$, because aluminium does trigger this response (Achary et al. , 2012). Moreover, we observed that actin remodelling in response to aluminium can be suppressed by diphenylene iodonium, indicative of RboH activity as transducing event (Figure 2 ). While in case of a real pathogen attack apoplastic oxidative burst is accompanied by calcium influx, this does not seem to be the case for aluminium (Suppl. Figure S3 ). A parsimonious hypothesis would therefore assume that calcium influx as a second signal is required to activate programmed cell death.

Calling for a role of salicylic acid signalling also in basal immunity

The pathway activated by aluminium activates some features that, in grapevine cells, usually are associated with cell-death related immunity, such as actin remodelling, or activation of RboH (Chang et al., 2015). On the other side aluminium die not induce cell death. We wondered, therefore, to what extent this would be reflected in activation of salicylic acid signalling, as salicylic acid is associated with defence against biotrophic pathogens, i.e. a condition, where cell-death related defence would provide an evolutionary advantage, while PTI is associated with jasmonate signalling (reviewed in Glazebrook, 2005). Also for grapevine cells, jasmonate signalling seems to be exclusively linked to PTI (Chang et al., 2017). Surprisingly, aluminium not only induced the transcripts for phytoalexin genes, but also activated isochorismate synthase, a key enzyme of salicyclic-acid biosynthesis (Figure 5B ), as well as the salicylic-acid responsive PR1 (Figure 5A ). In both cases, the induction by aluminium can be inhibited by Latrunculin $\mathrm{B}$, providing evidence for the involvement of the actin-dependent pathway. A link between actin and salicylic-acid signalling is apparently not confined to grapevine: In tobacco leaves, the marker genes PR1 and PR2 were significantly increased by treatment with cytochalasins (Kobayashi \& Kobayashi, 2007). A similar effect could be observed in Arabidopsis seedlings, when they were treated with actin filaments drugs latrunculin B and cytochalasin 
E. Here, the expression of PR1 , PR2 and WRKY38 was increased after 6 h (Matoušková et al., 2014), mimicking the patterns seen for salicylic acid.

The co-existence of salicylic-acid signalling with a defence pattern not accompanied by programmed cell death, indicates that salicylic acid, contradicting the current prevailing concept, can be recruited for basal immunity. Also this conclusion is in line with previous observations, where salicylic acid can activate a specific stilbene synthase promoter allele from a wild Chinese grapevine species, $V$. pseudoreticulata(Jiao et al., 2016).

What is the relationship of aluminium-triggered signalling with basal defence?

The fact that SA signalling can be activated by aluminium and is acting in the context of basal immunity provokes a new question: Is it possible to define the event, where the actin-dependent pathway merges with the signalling deployed in the context of PTI? The operational criterion for an event located downstream of this merging point would be that inhibition of this event would block both, gene expression induced by flg22, and gene expression induced by aluminium. It seems that MAPK signalling meets this criterion, because treatment of grapevine cells with the MAPK inhibitor PD98059 can fully suppress the induction of all three tested genes involved in phytoalexin synthesis (i.e. $P A L, R S$, and $S T S$, Figure 6A ), and the same was observed for the induction of STS by both flg22 and by harpin (Chang \& Nick, 2012). This inhibitor acts at the MAPKK level of the cascade (Cohen, 1997). Specifically for defence signalling, MKK1 has been suggested as target (Mészáros et al., 2006). Thus, at the second tier of MAPK signalling, flg22triggered, harpin-triggered, and aluminium-triggered signal transduction seem to be confluent already. Since aluminium, in contrast to flg22, fails to induce extracellular alkalinisation (Suppl. Figure S3 ), the merging point must be downstream of calcium influx. Furthermore, it must be located downstream of actin, since Latrunculin B can block the induction of gene expression by aluminium (Figure 3A ).

The fact that aluminium activates a key gene of SA synthesis (isochorismate synthase) and a marker gene for $\mathrm{SA}$ response (PR1) does not mean that $\mathrm{SA}$ is part of the signalling responsible for the activation of phytoalexin synthesis genes. This is rather unlikely, due to the time course. However, SA might act in a feedback loop contributing to the persistence of defence. A straightforward mechanism would be the accumulation of intracellular ROS. The original idea that this might be caused by direct inhibition of catalase through salicylic acid (Chen et al., 1993; Klessig et al. , 2019) has meanwhile been discarded after decades of controversy, and replaced by a model, where SA is acting through a signalling network that regulates expression and activities of the enzymatic and non-enzymatic redox homeostasis (for a recent review see Klessig et al. , 2019). The link with actin might come through phospholipase D: by binding to phospholipids, the substrates of phospholipid D, aluminium inhibits the formation of phosphatidic acids, as concluded from the fact that exogenous phosphatidic acids can mitigate the effect of aluminium in Arabidopsis seedlings (Matoušková et al., 2014).

There is a seemingly paradox observation, though: In our study, several of the genes that are activated by $\mathrm{Al}^{3+}$, are also activated by Latrunculin B significantly to the same extent. While the combination of $\mathrm{Al}^{3+}$ and Latrunculin B eliminates this activation. Interestingly, the transcription factor MYB14 does not show this activation by Latrunculin B. Although it appears paradox that two compounds that act antagonistically upon actin, should exert the same effect on gene activation, this is not the first time that this has been reported for phytoalexin synthesis genes in grapevine (Qiao et al., 2010). Also for MAPK signalling, the activation of the MAP kinase SIMK by both Latrunculin B and Jaspaklinolide has revealed a similar paradox (Šamaj et al. , 2002). Activation of signalling by elimination of actin could be achieved by releasing an activator that had been tethered to actin, as it has been proposed for the MAP kinases SIMK and SAMK (Šamaj et al. , 2002; Sangwan et al. , 2002). How signalling might be stimulated by suppression of actin turnover, is more challenging to explain. One link might be Capping Protein that can either be sequestered to the plasma membrane by phosphatidic acids, the products of the phospholipase D signalling hub, or block the elongation of actin filaments by blocking the barbed end ( $\mathrm{Li}$ et al. , 2015). Stabilisation of actin might repartition Capping Protein from the membrane towards bundling actin cables, such that phosphatidic acid would be released for other signalling functions, such as activation of the Respiratory burst oxidase Homologue 
(Eggenberger et al. , 2017), leading to a self-amplifying signalling loop between actin and reactive oxygen species. The fact that some genes $(P A L, R S, S T S)$ show both, the activation by $\mathrm{Al}^{3+}$ and by Latrunculin $\mathrm{B}$, while others (MYB14 ) are not activated by Latrunculin $\mathrm{B}$, indicates that the signal chains initiating from actin (one possibly through MAPK signalling, the other possibly through phosphatidic acid signalling) might be activated differentially and exert differing impact on different target genes.

Outlook: From the cell culture to the vineyard - routes towards application.

The induction of oxidative burst (Figure 7C ), transcripts for MYB14 and RS (Figure 7A ), and accumulation of the bioactive phytoalexin, trans -resveratrol (Figure 7B ) by $\mathrm{Al}^{3+}$ in leaf discs of grapevine indicates that the signalling chain discussed above is active not only in suspension cells but in planta as well. Even genotype-dependent differences in the response amplitude between the more responsive sylvestrisgenotype Hö29 and the less responsive vinifera genotype Augster Weiss seen in earlier studies (Duan et al. , 2015; 2016) are preserved. The differences seen on the level of phytoalexins synthesis transcripts $(R S)$ or the specific transcription factor triggering these genes (MYB14) are preceded by corresponding differences in the abundance of MDA. This indicates that the differential response of the two genotypes, which is also seen for UVC or the Oomycete pathogenPlasmopara viticola (Duan et al. , 2015) is caused far upstream, possibly at the abundance or activation of RboH. Our findings also opens a new interpretation for the mode of action of Al-based compounds acting against Oomycete pathogens (Dercks \& Buchenauer, 1987). Rather than acting through interfering with the pathogen itself, such compounds might act to stimulate or prime the immunity of the host. Especially for P. viticola, the causative agent of Downy Mildew of Grapevine, this would open new avenues for plant protection. Rather than the need to rely on the very short time window, where the infecting zoospore still has not entered the stoma (leading to the excessive fungicide load characteristic for viticulture), a prophylactic treatment would be feasible, where the host, rather the pathogen is addressed.

\section{Acknowledgements}

This work was supported by Interreg Upper Rhine (Vitifutur) co-financed by the European Union/European Regional Development Fund (ERDF), the German Federal Agency for Agriculture (Programme for Sustainable Agriculture, BÖLN), and by a fellowship from the China Scholarship Council to Dong Duan and Ruipu Wang. Hao Wang is acknowledged for kindly providing the FABD2-GFP actin-marker line.

\section{Reference}

Achary, V. M. M., Parinandi, N. L., \& Panda, B. B. (2012). Aluminum induces oxidative burst, cell wall NADH peroxidase activity, and DNA damage in root cells of Allium cepa L. Environmental and Molecular Mutagenesis , 53(7), 550-560. https://doi.org /10. 1002 /em.21719.

Ahad, A., \& Nick, P. (2007). Actin is bundled in activation-tagged tobacco mutants that tolerate aluminum. Planta , 225(2), 451-68. https://doi.org/10.1007/s00425-006-0359-0.

Akaberi, S., Wang, H., Claudel, P., Riemann, M., Hause, B., Hugueney, P., \& Nick, P. (2018). Grapevine Fatty Acid Hydroperoxide Lyase Generates Actin-Disrupting Volatiles and Promotes Defence-Related Cell Death.Journal of experimental botany , 69(12), 2883- 2896. https://doi.org/10.1093/jxb/ery133.

Bigeard, J., Colcombet, J., \& Heribert, H. (2015). Signaling mechanisms in pattern-triggered immunity (PTI). Molecular Plant , 8(4), 521-539. https://doi.org/10.1016/j.molp.2014. 12.022.

Chang, X. L., Heene, E., Qiao, F., \& Nick, P. (2011) The phytoalexin resveratrol regulates the initiation of hypersensitive cell death in Vitis cell. PLoS One , 6(10), e26405. https://doi. org/10.1371/journal.pone.0026405.

Chang, X. L., \& Nick, P. (2012). Defence Signalling Triggered by Flg22 and Harpin Is Integrated into a Different Stilbene Output .in VitisCells. PLoS One , 7 (7), e404467. https://doi.org/10.1371/journal.pone.0040446.

Chang, X. L., Riemann, M., Liu, Q., \& Nick, P. (2015). Actin as deathly switch? How auxin can suppress cell-death related defence. PLoS One, 10 (5), e0125498. https://doi.org/10. 1371 /journal.pone.0125498. 
Chang, X. L., Seo, M., Takebayashi, Y., Kamiya, Y., Riemann, M., \& Nick, P. (2017) Jasmonates are induced by the PAMP flg22 but not the cell death-inducing elicitor Harpin in Vitis rupestris .Protoplasma, 254(1), 271-283. https://doi.org/10.1007 /s00709-016-0941-7.

Chen, Z., Silva, H., \& Klessig, D. F. (1993) Active oxygen species in the induction of plant systemic acquired resistance by salicylic acid.Science, 262(5141), 1883-1886. https://doi. org/10.1126/science.8266079.

Chen, Z. X., Zheng, Z. Y., Huang, J. L., Lai, Z. B., \& Fan, B. F. (2009). Biosynthesis of salicylic acid in plants. Plant Signaling \& Behavior . 4(6), 493-496. https://doi.org/10. 4161 /psb.4.6.8392.

Cohen, P. (1997) The search for physiological substrates of MAP and SAP kinases in mammalian cells. Trends in Cell Biology , 7(9), 353- 361. https://doi.org/10.1016 /S0962-8924(97)01105-7.

Dercks, W., \& Buchenauer, H. (1987) Comparative studies on the mode of action of aluminium ethyl phosphite in four Phytophthora species.Crop Protection , 6(2), 82-89. https://doi.org/10.1016/0261-2194(87)90104-9.

Duan, D., Halter, D., Baltenweck, R., Tisch, C., Tröster, V., Kortekamp, A., Hugueney, P., \& Nick, P. (2015) Genetic diversity of stilbene metabolism in Vitis sylvestris . Journal of Experimental Botany ,66(11), 3243-3257. https://doi.org/10.1093/jxb/erv137.

Duan, D., Fischer, S., Merz, P., Bogs, J., Riemann, M., \& Nick, P. (2016). An ancestral allele of grapevine transcription factor MYB14 promotes plant defence. Journal of Experimental Botany , 67(6), 1795-1804. https://doi.org/10.1093/jxb/erv569.

Eggenberger, K., Sanyal, P., Hundt, S., Wadhwani, P., Ulrich, A. S., \& Nick, P. (2017). Challenge Integrity: The Cell-Penetrating Peptide BP100 Interferes with the Auxin-Actin Oscillator. Plant and Cell Physiology , 58(1), 71-85. https://doi.org/10. 1093/pcp/pcw161

Franklin-Tong, V., \& Gourlay, C. W. (2008). A role for actin in regulating apoptosis/programmed cell death: evidence spanning yeast, plants and animals. Biochemical Journal , 413(3), 389-404. https://doi.org/10.1042/BJ20080320

Felix, G., Regenass, M., \& Boller, T. (1993). Specific perception of subnanomolar concentrations of chitin fragments by tomato cells: induction of extracellular alkalinization, changes in protein phosphorylation, and establishment of a refractory state. The Plant Journal , 4(2), 307-316. https://doi.org/10.1046/j. 1365313X.1993. 04020307.x

Gaff, D. F., \& Okong'o-ogola, O. (1971). The Use of Non-permeating Pigments for Testing the Survival of Cells. Journal of Experimental Botany, 22(3), 756-758. https://doi.org/10. 1093 /jxb/22.3.756.

Glazebrook, J. (2005) Contrasting mechanisms of defense against biotrophic and necrotrophic pathogens. Annual Review of Phytopathology , 43, 205-227. https://doi.org/10.1146/ annurev. phyto.43.040204.135923

Gómez-Gómez, L., \& Boller, T. (2002) Flagellin perception: a paradigm for innate immunity. Trends in Plant Science , 7(6), 251-256. https://doi.org/10.1016/s1360-1385(02)02261-6.

Gourlay, C. W., \& Ayscough, K. R. (2005) The actin cytoskeleton: a key regulator of apoptosis and ageing? Nature reviews Molecular cell biology , 6(7), 583-589. https://doi. org/10.1038/nrm1682.

Guan, X., Buchholz, G., \& Nick, P.(2013). The cytoskeleton is disrupted by the bacterial effector HrpZ, but not by the bacterial PAMP flg22, in tobacco BY-2 cells. Journal of Experimental Botany, 64(7), 1805-1816. https://doi.org/ 10.1093/jxb/ert042

Heath, R. L., \& Packer, L. (1968). Photoperoxidation in isolated chloroplasts: I. Kinetics and stoichiometry of fatty acid peroxidation.Archives of biochemistry and biophysics , 125(1), 189-198. https://doi.org/10.1016/0003-9861(68)90654-1

Hodgson, R. A. J., \& Raison, J. K. (1991) Lipid peroxidation and superoxide dismutase activity 
in relation to photoinhibition induced by chilling in moderate light. Planta , 185(2), 215-219. https://doi.org/10.1007/BF00194063

Höll, J., Vannozzi, A., Czemmel, S., D’Onofrio, C., Walker, A. R., Rausch, T., Lucchin, M., Boss, P. K., Dry, I. B., \& Bogs, J. (2013) The R2R3-MYB transcription factors MYB14 and MYB15 regulate stilbene biosynthesis in Vitis vinifera. The Plant Cell , 25(10), 4135-4149. https://doi.org/10.1105/tpc.113.117127

Ikegawa, S., Oohashi, J., Murao, N., \& Goto, J. (2000). A method for the determination of the hepatic enzyme activity catalyzing bile acid acyl glucuronide formation by high-performance liquid chromatography with pulsed amperometric detection. Biomedical chromatography: BMC , 14(3), 144-150. https://doi.org/10.1002/ 1099-0801(200005)14:3<144::AID-BMC939>3.0.CO;2-K

Jiao, Y., Xu, W., Duan, D., Wang, Y., \& Nick, P. (2016) A stilbene synthase allele from a Chinese wild grapevine recruits salicylic acid signalling for efficient defence. Journal of Experimental Botany , 67(19), 5841-5856. https://doi.org/10.1093/jxb/erw351.

Jones, J. D. G., \& Dang, J. L. (2006). The plant immune system. Nature , 444(7117), 323-329. https://doi.org/10.1038/nature05286

Klessig, D. F., Choi, H. W. \& Dempsey, D. A. (2019) Systemic Acquired Resistance and Salicylic Acid: Past, Present, and Future. Molecular Plant Microbe Interactions , 31(9), 871-888. https://doi.org/10.1094/MPMI03-18-0067-CR.

Kobayashi, Y., \& Kobayashi, I. (2007). Depolymerization of the actin cytoskeleton induces defense responses in tobacco plants. Journal of General Plant Pathology, 73(5), 360-364. https://doi.org/10.1007/s10327-0070029-5

Li, J. J., Blanchoin, L., \& Staiger, C. J. (2015). Signaling to Actin Stochastic Dynamics. Annual Review of Plant Biology . 66, 415-440. https://doi.org/10.1146/annurev- arplant-050213-040327.

Liu, L. J., Sonbol, F., Huot, B., Gu, Y. N., Withers, J., Mwimba, M., Yao, J., He, S. Y., \& Dong, X. N. (2016). Salicylic acid receptors activate jasmonic acid signalling through a non-canonical pathway to promote effector-triggered immunity. Nature Communications ,7 , 13099. https://doi.org/10.1038/ncomms13099.

Liu, Y., \& He,C. (2017) A review of redox signaling and the control of MAP kinase pathway in plants. Redox Biology , 11, 192-204. https://doi. 10.1016/j.redox.2016.12.009.

Maisch, J., Fiserová, J., Fischer, L., \& Nick, P. (2009). Tobacco Arp3 is localized to actin-nucleating sites in vivo. Journal of experimental Botany , 60(2), 603-614. https:// doi. org/10.1093/jxb/ern307.

Matoušková, J., Janda, M., Fišer, R., Šašek, V., Kocourková, D., Burketová, L., Dušková, J., Martinec, J., \& Valentová, O. (2014). Changes in actin dynamics are involved in salicylic acid signaling pathway. Plant Science, 223, 36-44. https://doi.org/10.1016 /j.plantsci.2014.03.002

Mészáros, T., Helfer, A., Hatzimasoura, E., Magyar, Z., Serazetdinova, L., Rios, G., Bardóczy, V., Teige, M., Koncz, C., Peck, S. \& Bögre, L. (2006) The Arabidopsis MAP kinase kinase MKK1 participates in defence responses to the bacterial elicitor flagellin. The Plant Journal , 48(4), 485-498. https://doi.org/10.1111/j.1365313X.2006.02888.x.

Mittler, R. (2006). Abiotic stress, the field environment and stress combination. Trends in Plant Sciences , 11(1), 15-19. https://doi.org/10.1016/j.tplants.2005.11.002.

Panda, S. K., Baluška, F., \& Matsumoto, H. (2009). Aluminum stress signaling in plants. Plant Signaling \& Behavior , 4(7), 592-597. https://doi.org/10.4161/psb.4.7.8903

Qiao, F., Chang, X. L., \& Nick, P. (2010). The cytoskeleton enhances gene expression in the response to the Harpin elicitor in grapevine.Journal of Experimental Botany , 61(14), 4021-4031. https://doi.org/10.1093/jxb/erq221. 
Ramirez-Prado, J. S., Abulfaraj, A. A., Rayapuram, N., Benhamed, M., \& Hirt, H. (2018). Plant Immunity: From Signaling to Epigenetic Control of Defense.Trends in Plant Science , 23(9), 833-844. https://doi.org/10.1016/j.tplants.2018.06.004.

Rentel, M. C., Lecourieux, D., Ouaked, F., Usher, S. L., Petersen, L., Okamoto, H., Knight, H., Peck, S. C., Grierson, C. S., Hirt, H. \& Knight, M. R. (2004). OXI1 kinase is necessary for oxidative burst-mediated signalling in Arabidopsis . Nature , 427(6977), 858-861. https://doi. org/10.1038/nature02353.

Šamaj, J., Ovecká, M., Hlavacká, A., Lecourieux, F., Meskiene, I., Lichtscheidl, I., Lenart, P., Salaj, J., Volkmann, D., Bögre, L., Baluska, F., \& Hirt, H. (2002). Involvement of the mitogen-activated protein kinase SIMK in regulation of root hair tip growth. EMBO Journal ,21(13), 3296-3306. https://doi.org/10.1049/ipmap:19951763.

Šamaj, J., Baluška, F. \& Hirt, H. (2004) From signal to cell polarity: mitogen-activated protein kinases as sensors and effectors of cytoskeleton dynamicity. Journal of Experimental Botany , 55(395), 189-198. https://doi.org/10.1093/jxb/erh012.

Sangwan, V., Orvar, B. L, Beyerly, J., Hirt, H., \& Dhindsa R. S. (2002). Opposite changes in membrane fluidity mimic cold and heat stress activation of distinct plant MAP kinase pathways. The Plant Journal, 31(5), 629-638. https://doi.org/10.1046/j.1365- 313x. 2002.01384.x.

Schwarzerová, K., Zelenková, S., Nick, P., \& Opatrný, Z. (2002). Aluminum-induced rapid changes in the microtubular cytoskeleton of tobacco cell lines. Plant and Cell Physiology , 43(2), 207-216. https://doi.org/10.1093/pcp/pcf028.

Seibicke, T., Rügner, A., Neuhaus, G., Kassemeyer, H., \& Buchholz, G. (2002). Assay system to screen for compounds inducing PR-gene expression in grapevine (Vitis spec.). Induced Resistance in Plants against Insects and Diseases , 25(6), 63-66. http://orgprints. org/14635/.

Smertenko, A., \& Franklin-Tong, V. E. (2011). Organisation and regulation of the cytoskeleton in plant programmed cell death. Cell Death \& Differentiation , 18(8), 1263-1270. https://doi.org/10.1038/cdd.2011.39.

Tsuda, K., \& Katagiri, F. (2010). Comparing signaling mechanisms engaged in patterntriggered and effector-triggered immunity.Current Opinion in Plant Biology , 13(4), 459-465. https://doi.org/10.1016/j.pbi.2010.04.006.

Waller, F., \& Nick, P. (1997). Response of actin microfilaments during phytochrome-controlled growth of maize seedlings. Protoplasma , 200(3-4), 154-162. https://doi.org/10.1007/BF01283291.

Waller, F., Riemann, M., \& Nick, P. (2002). A role for actin-driven secretion in auxin-induced growth. Protoplasma , 219(1-2), 72-81. https://doi.org/10.1007/s007 090200007.

Wasteneys, G. O., \& Yang, Z. B. (2004). New views on the plant cytoskeleton. Plant Physiology , 136(4), 3884-3891. https://doi.org/10.1104/pp.104.900133.

Withers, J., \& Dong, X. N. (2017). Post-translational regulation of plant immunity. Current Opinion in Plant Biology , 38, 124-132. https://doi.org/10.1016/j.pbi.2017.05.004. 

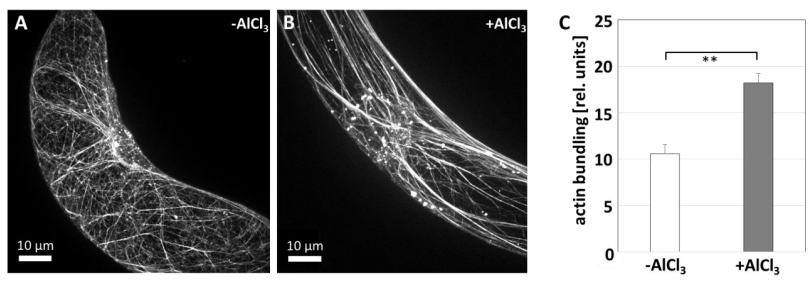

FIGURE 1 Response of actin filaments to aluminium ions. Representative images of grapevine cells expressing the actin marker fimbrin actin-binding domain 2 in fusion with GFP in the absence (A) or in the presence (B) of $\mathrm{AlCl}_{3}(200 \mu \mathrm{M}, 2 \mathrm{~h})$. (C) Quantification of the actin responses. Asterisks indicate significant differences with ** $\mathrm{P}<0.01$. 

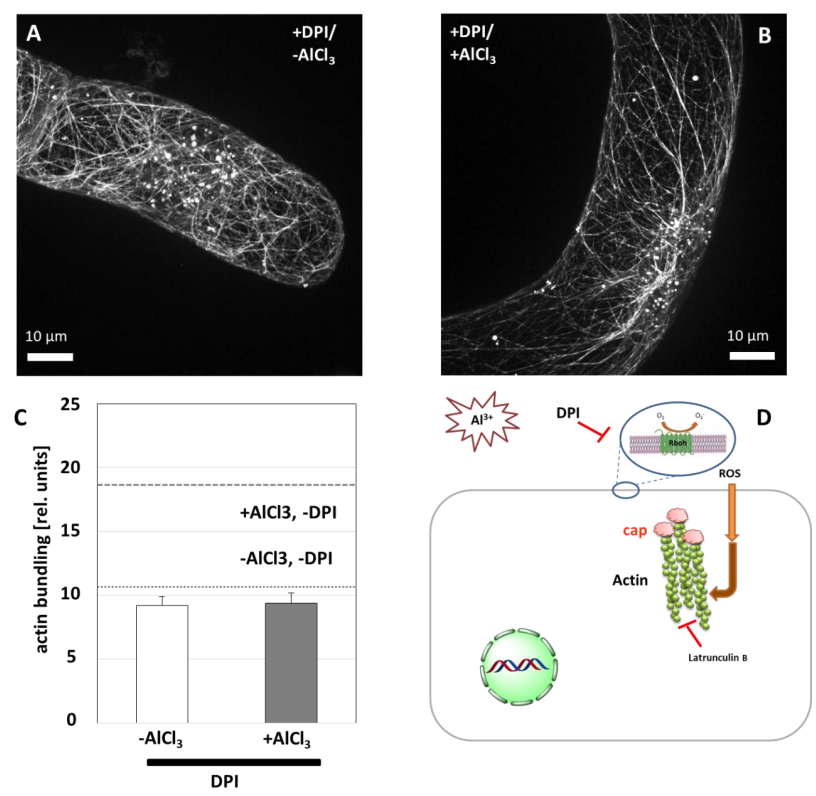

FIGURE 2 Effect of diphenylene iodonium (DPI) pretreatment (30 min, $20 \mu \mathrm{M}$ ) on the aluminium-induced response of actin filaments. Representative images of grapevine cells expressing the actin marker fimbrin actin-binding domain 2 in fusion with GFP in the absence (A) or in the presence (B) of $\mathrm{AlCl}_{3}(200 \mu \mathrm{M}, 2 \mathrm{~h})$ are shown. (C) Quantification of the actin response. The dashed line gives the amplitude of actin bundling in response to $\mathrm{AlCl}_{3}$ alone, in the absence of DPI, the dotted line the value seen in untreated controls for comparison (see FIGURE 1). (D) Working hypothesis tested by this experiment. 
A

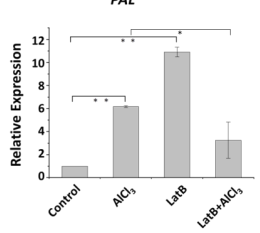

RS

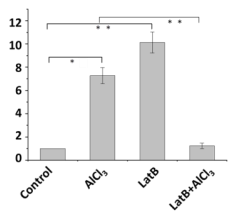

B

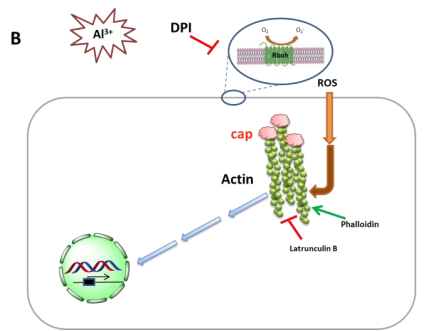

STS

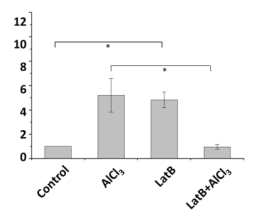

FIGURE 3 (A) Response of steady-state transcript levels for phenylammonium lyase $(P A L)$, the resveratrol synthase subpopulation of the stilbene synthase family $(R S)$, and the canonical stilbene synthases (STS) to either $200 \mu \mathrm{M} \mathrm{AlCl} 3(2 \mathrm{~h}), 1 \mu \mathrm{M}$ Latrunculin B (LatB, $2 \mathrm{~h}$ ), or a combination of Latrunculin B (30 min) pretreatment followed by $\mathrm{AlCl}_{3}(2 \mathrm{~h})$ treatment. Data represent mean values and standard errors from three independent experimental series with three technical replications for each biological replicate. Transcript levels are calibrated to $E F-1 \alpha$ as internal standard. Asterisks indicate significant differences with* $\mathrm{P}<0.05$ and $* * \mathrm{P}<0.01$. (B) Working hypothesis tested by this experiment. 
MYB 14

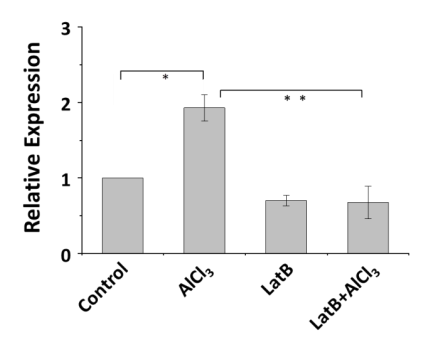

FIGURE 4 Response of steady-state transcript levels for MYB14 to either $200 \mu \mathrm{M}$ $\mathrm{AlCl}_{3}(2 \mathrm{~h}), 1 \mu \mathrm{M}$ Latrunculin B (LatB, $2 \mathrm{~h}$ ), or a combination of Latrunculin B (LatB, $30 \mathrm{~min}$ ) pretreatment followed by $\mathrm{AlCl}_{3}(2 \mathrm{~h})$ treatment. Data represent mean values and standard errors from three independent experimental series with three technical replications for each biological replicate. Transcript levels are calibrated to $E F-1 \alpha$ as internal standard. Asterisks indicate significant differences with* $\mathrm{P}<0.05$ and $* *$ $\mathrm{P}<0.01$. 

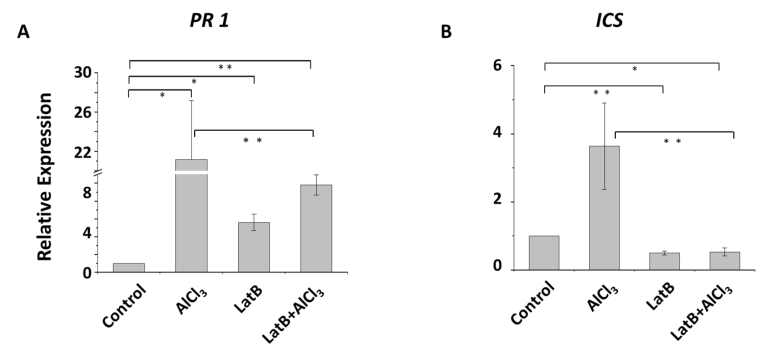

FIGURE 5 Response of steady-state transcript levels for pathogenesis related 1 (PR1, A), and isochorismate synthase (ICS, B), to either $200 \mu \mathrm{M} \mathrm{AlCl} \mathrm{Al}_{3}(2 \mathrm{~h}), 1 \mu \mathrm{M}$ Latrunculin B (LatB, 2 h), or a combination of Latrunculin B (LatB, 30 min) pretreatment followed by treatmen with $\mathrm{AlCl}_{3}(2 \mathrm{~h})$. Data represent mean values and standard errors from three independent experimental series with three technical replications for each biological replicate. Transcript levels are calibrated to $E F-1 \alpha$ as internal standard. Asterisks indicate significant differences with* $\mathrm{P}<0.05$ and $* *$ $\mathrm{P}<0.01$. 


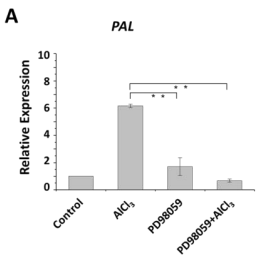

+PD98059/-AlCl 3

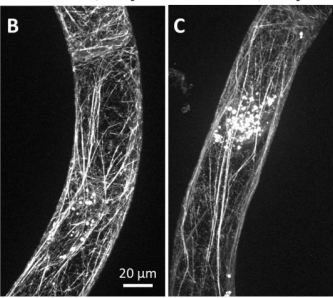

E

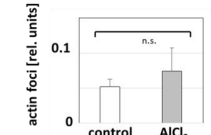

RS

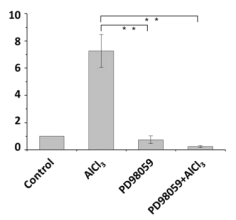

STS

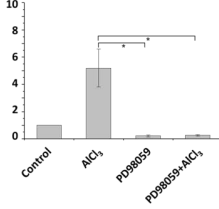

D

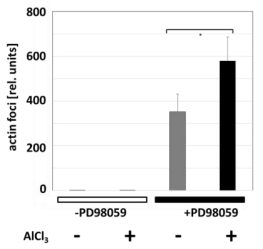

$\mathrm{AlCl}_{3}-++-$

$\mathbf{F}$
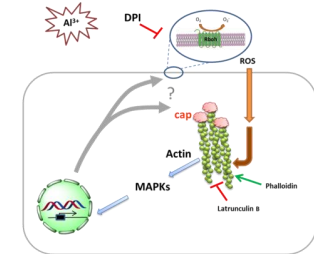

FIGURE 6 (A) Response of steady-state transcript levels for phenylammonium lyase $(P A L)$, the resveratrol synthase subpopulation of the stilbene synthase family $(R S)$, and the canonical stilbene synthases (STS) to either $200 \mu \mathrm{M} \mathrm{AlCl}(2 \mathrm{~h}), 50 \mu \mathrm{M}$ PD98059 (2 h), or a combination of PD98059 (30 min) pretreatment followed by $\mathrm{AlCl}_{3}(2 \mathrm{~h})$ treatment. Data represent mean values and standard errors from three independent experimental series with three technical replications for each biological replicate. Transcript levels are calibrated to $E F-1 \alpha$ as internal standard. Asterisks indicate significant differences with* $\mathrm{P}<0.05$ and $* * \mathrm{P}<0.01$. (B) and $(\mathrm{C})$ are the responses of actin filaments to -/+aluminum $(200 \mu \mathrm{M}, 2 \mathrm{~h})$ in presence of PD98059 $(50 \mu \mathrm{M})$. Representative images of grapevine cells expressing the actin marker fimbrin actin-binding domain 2 in fusion with GFP. (D) and (E) are the quantification of formation of actin foci. Due to the response is much weaker in absence of PD98059 in (D), values are replotted in (E) with a different scale. (F) Working hypothesis tested by this experiment. 
A

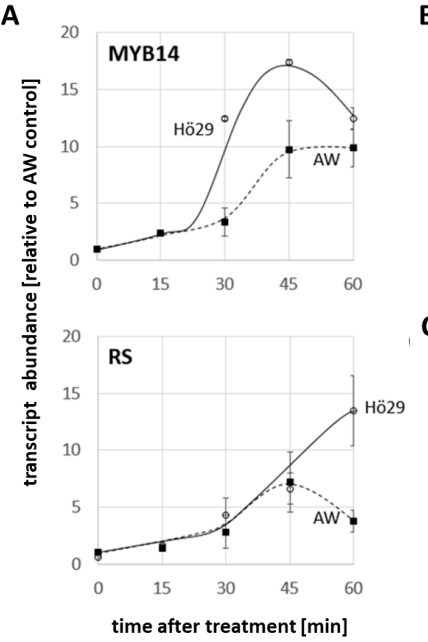

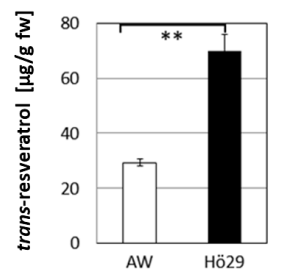

C

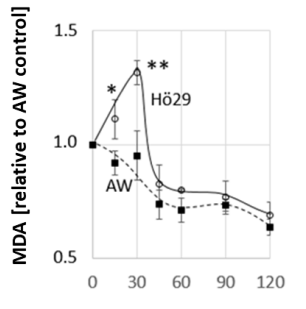

time after treatment [min]

FIGURE 7 Responses of grapevine leaf discs to aluminium (1\%) in the high-stilbene chemovar V. sylvestris Hö 29 and the low-stilbene chemovar V. vinifera Augster Weiss (AW). (A) Time course for steady-state transcript levels of MYB14 and RS. (B) Contents of the active stilbene trans-resveratrol measured $24 \mathrm{~h}$ after the treatment with aluminium. (C) Abundance of the lipid peroxidation product malone dialdehyde (MDA) over time following treatment with aluminium. Data represent mean values and standard errors from three independent experimental series with three technical replications for each biological replicate. Asterisks indicate significant differences with* $\mathrm{P}<0.05$ and $* * \mathrm{P}<0.01$. 